Rev. Elev. Méd. Vét. Pays trop., 1968, 21, 4 (443-449).

\title{
Enquête sur l'infection à virus parainfluenza 3 chez le dromadaire tchadien
}

\author{
por Y. MAURICE * R. QUEVAL * J. F. BARES **
}

\begin{abstract}
RÉSUME
Les auteurs ont étudıé l'incidence de l'ınfection à virus paraınfluenza $3 \mathrm{chez}$ le dromadaire du Tchad, en analysant par la technique d'inhibition de l'hémagglutination 559 sérums de cette espèce. Les résultats obtenus montrent que I'infection cameline à vırus parainfluenza 3 est largement répandue dans les régıons sahéliennes de ce pays. Il est vraisemblable que l'infection puisse évolver sans forme aiguë ef de façon épizootique à l'ıntérieur d'un même troupeau.

Les sérums de dromadares ne semblent pas contenır d'inhıbiteurs non spécifiques de l'hémagglutination par le virus parainfluenza 3. Les sérums qui sont négatifs à ce test d'ınhibitıon ne correspondent pas à des animaux hypo ou agammaglobulinémıques.
\end{abstract}

Myxovirus parainfluenza 3 a été identıfié chez l'homme, les bovins ef les chevaux atteints d'affections respiratoires. Différentes enquêtes sérologiques ont mis en évidence la présence d'anticorps spécifiques chez les bovins sur les continents américain ef européen, au Japon ef récemment au Tchad.

On sait que Myxovirus parainfluenza 3 a été incriminé dans la maladie des transports («shipping fever») des bovins qui se traduit, dans la forme bénigne, par des atteintes des vaies respiratoires supérieures de type catarrhal, et dans la forme grave, par des pneumonies et des broncho-pneumonies. II étaif intéressant de savoir si ce virus pouvalt intervenir dans les affections groupées sous le vocable «complexe des maladies respiratoires du dromadaire $»$. C'est dans cet esprit qu'a été effectuée cette enquête sérologique à partır des sérums de dromadaires de différentes régions du Tchad.

(*) I. E. M. V. T. Laboratoire de recherches vérérinaires de Farcha, Fort-Lamy, Tchad.

(**) Service de l'Elevage du Tchad.

\section{MATÉRIEL ET MÉTHODE}

1) Sérums : les 559 sérums étudiés proviennent de dromadaires qui ont été présentés individuellement à des postes vétérinaires pour $y$ subir un traitement (antitrypanosomien en général), ou de sujets appartenant aux pelotons méharistes des régions nord-sahéliennes de Nokou, Ziguey et Moussoro. L'orıgine géographique des sérums prélevés correspond, en gros, à la répartition des dromadaires au Tchad, c'est-à-dire à la région située au Nord de l'isohyète $500 \mathrm{~mm}$. Les régions de Nokou, Ziguey, Mao, Moussoro, au Kanem, celle d'Abéché au Ouaddaı ont été intéressées par cette enquête. Quelques sérums ont été prélevés à Fort-Lamy même, mais ils correspondent à des animaux du Kanem. La répartition exacte de ces sérums est la suivante:

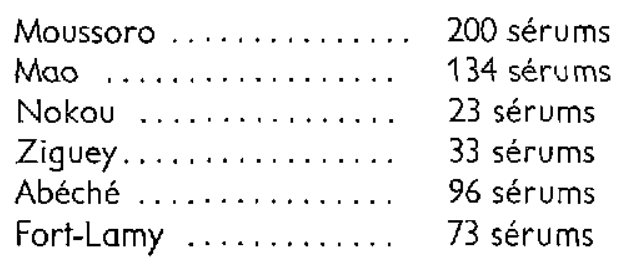




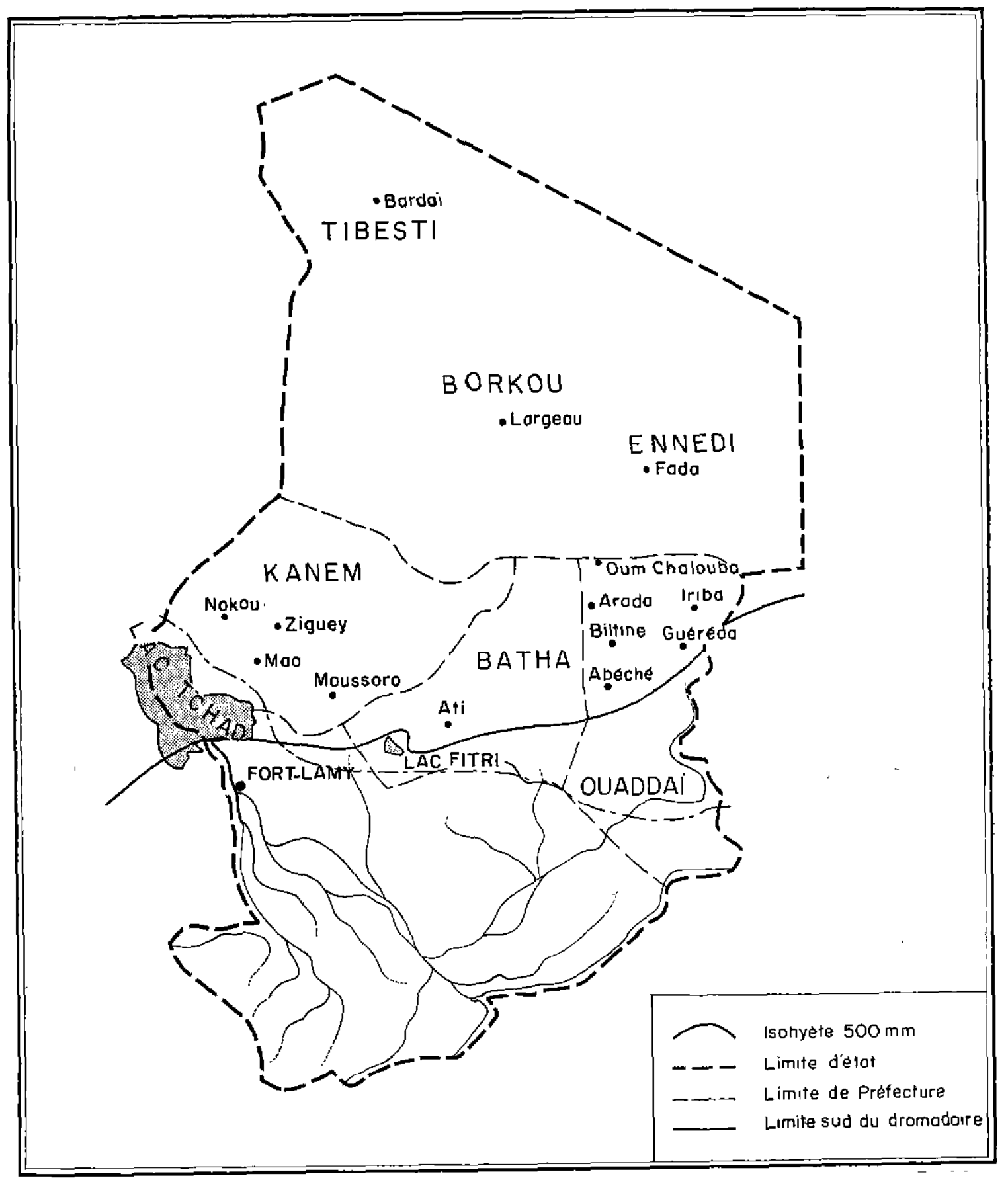


Aucun antiseptique n'a été ajouté aux sérums ; tous ont été congelés et décongelés à plusieurs reprises, parce que déjà utılisés pour d'autres enquêtes.

2) Technique de la réaction d'inhibition de I'hémagglutination due au virus parainfluenza 3 :

a) préparation de l'hémagglutinine : c'est exactement la même que celle exposée à l'occasion d'une enquête similaire sur les bovins d'Afrique Centrale par PROVOST et coll. (11) ;

b) technique d'inhibition : si la présence d'inhibiteurs non spécifiques de l'hémagglutination parainfluenza 3 est exceptionnelle chez les bovins (DAWSON) (2), (KETLER et coll.) (8), (HAMPARIAN et coll.) (5), il paraissait nécessaire de vérifier s'il en était de même chez le dromadare, d'autant plus que, récemment, FAYE, CHARTON et LE GAYEC (3) ont mis en évidence, dans les sérums d'ovins des anticorps inhibant cette hémagglutination. Ainsi, un lot de 78 sérums de dromadaires inhibant l'hémagglutination par ce virus à un taux $>1 / 10$, a subi les traitements suivants, seuls ou combinés :

a-Célimination des hétéroagglutinınes par contact d'ume heure d̀ $4^{\circ} \mathrm{C}$ entre sérum et hématies de cobaye, puis séparation de ces hématies par centrifugation lente :

$\beta$-élimination de l'inhıbıteur type $\mathrm{CHU}$ thermolabile par chauffage $30 \mathrm{mn}$ ò $56^{\circ} \mathrm{C}$;

$\gamma$-élimination de l'ınhibiteur de FRANCIS éventuellement présent par contact $20 \mathrm{mn}$ à la température du laboratore avec une suspension à 25 p. 100 de kaolin en tampon salé boraté, ef centrifugation de $30 \mathrm{mn}$ à $1.000 \mathrm{~g}$, la dilution finale du sérum étant à $1: 10$.

Les sérums examinés sont dilués directement en sérum physiologique du 1/10 au 1/640, et ceux n'ayant pas subi le traitement au kaolin sont également dilués au $1 / 2,1 / 4$ et $1 / 8$. On mélange à $0,2 \mathrm{ml}$ des dilutions de chaque sérum, $0,2 \mathrm{ml}$ d'hémagglutinine diluée en sérum physiologique pour contenir 4 unités hémagglutinantes par $0,2 \mathrm{ml}$ puis après un séjour d'une heure à $37^{\circ} \mathrm{C}$ on ajoute $0,2 \mathrm{ml}$ de la suspension d'hématies à 0,4 p. 100. On lit l'inhibition de l'hémagglutination lorsque les témoins de la suspension globulaire sont sédimentés. Les réactions sont effectuées dans des tubes de verre.
L'expérience préliminaire portant sur les 78 sérums ayant montré l'absence d'inhibiteurs non spécifiques de l'hémagglutination par le virus parainfluenza 3, les 481 sérums restant ont été analysés après avoir subi seulement les traitements $\alpha$ et $\beta$.

3) Electrophorèses : Elles ont été effectuées suivant la technique couramment utilisée à Farcha et rapportée par l'un de nous (12).

a) Protidémie. La détermination des protéines sériques a été réalisée de la façon suivante :

- dosage des protéines totales. La méthode de dosage des protéines employée est celle de GORNALL, BARDAWILL et DAVID, préconisée por FLEURY (4).

Cette méthode au biuret nécessite le calcul d'un coefficient de correction car, à côté des protéines, le sérum contient toujours en quantité variable d'autres substances bıurétogènes qui apportent dans les mesures des erreurs par excès.

- Dosage des globulines par précipitation avec le sulfate d'ammonium à saturation et dosage photométrique.

- Dosage des sérums albumines. Le poids des sérums albumines se calcule en faisant la différence entre le poids des protéınes totales et celui des sérums-globulines.

b) Analyse du protéinogramme. On a utilısé l'appareil à électrophorèse sur papier «JOUAN》 type CNRS. Les conditions expérimentales sont les suivantes :

- papier Arches 304,

- tompon de DURRUM de pH 8,6, de force ionique $0,05 \Gamma$,

— durée de l'électrophorèse : $2110 \mathrm{mn}$ sous une tension stabilisée de 400 volts.

- coloration au bleu de bromophénol.

- enregistrement photométrique semi-automatique,

- décomposition de l'électrophoregramme en courbes de Gauss,

- mesure des surfaces à l'aide du planimètre de Morin.

\section{RÉSULTATS}

1) Etude des inhibiteurs non spécifiques : Les combinaisons sulvantes ont été réalisées sur les 78 sérums étudiés. 
- sérum dont les inhibiteurs du type CHU et FRANCIS sont éliminés.

- sérum dont les inhibiteurs du type $\mathrm{CHU}$ et FRANCIS ainsi que les hétéroagglutinınes sont éliminés.

- sérum dont les inhibiteurs du type FRANCIS et les hétéroagglutinines sont éliminés.

- sérum dont les inhibiteurs du type $\mathrm{CHU}$ et les hétéroagglutinines sont éliminés.

- sérum brut, sans traitement.

Les résultats obtenus dans la réaction d'inhibition de l'hémagglutination avec le virus parainfluenza 3 avec chacune des 5 combinaisons réalisées pour chaque sérum sont compdrables. Il apparaît donc que les sérums de dromadaires ne contıennent pas d'inhibiteurs non spécifiques déceiables par ces méthodes. Certains sérums sont cependant justiciables de l'adsorption par les globules rouges de cobaye; en effet quelques agglutinations ont été observées avec des sérums non dilués.

2) Incidence générale des anticorps :

Les résultats globaux sont les suivants :

Nombre de sérums examinés : 559 ;

Nombre de sérums positifs: 555 soit 99,28 p. 100 ;
Nombre de sérums négatifs: 4 soit 0,72 p. 100.

La localisation géographique ef le pourcentage d'anticorps sont rapportés dans les tableaux I et II.

3) Comportement électrophorétique de quelques sérums négatifs, faiblement positifs et franchement positifs.

Comme ce fut le cas lors d'une enquête précédente (11) on pouvait se demander, étant donné le pourcentage élevé de sérums de dromadaires positifs, si les 4 sérums qui n'avaient pas d'anticorps décelables et ceux qui en possédaient à un titre faible $(1 / 2,1 / 4,1 / 8)$ n'entraient pas dans une catégorie d'animaux agamma ou hypogammaglobulinémiques (11). C'est paurquoi les 4 sérums négatifs et 23 sérums positifs à un titre inférieur à $1 / 10(1 / 2,1 / 4,1 / 8)$ ont été analysés et les résultats comparés à ceux des 30 sérums positifs à un titre supérieur ou égal à $1 / 10(1 / 10$ à $1 / 160)$.

Les résultats enregistrés avec ces trois catégories de sérums sont statistiquement comparables aux investigations réalisées lors d'une précédente enquête (13). La valeur moyenne des résultats obtenus est rapportée sur le tableau III, sur lequel sont également reportées les valeurs moyennes trouvées par d'autres auteurs.

TABLEAU N ${ }^{\circ} \mathrm{I}$

Pourcentage de sẻrums positlfs sulvant les localitês

\begin{tabular}{|c|c|c|}
\hline Localitêo & $\begin{array}{c}\text { Nombre de sérums } \\
\text { examinés }\end{array}$ & $\begin{array}{c}\text { Nombre de gérums positifs } \\
\text { et pourcentage }\end{array}$ \\
\hline $\begin{array}{l}\text { Moussoro } \\
\text { Lot } n^{*} 1 \\
\text { Lot n } 2^{*}\end{array}$ & $\begin{array}{r}137 \\
63\end{array}$ & $\begin{array}{cc}137 \text { solt } 100 & \text { P. } 100 \\
63 \text { solt } 100 & \text { " }\end{array}$ \\
\hline Mao & 134 & 131 soit 97,76 p. 100 \\
\hline Nokou ${ }^{+}$ & 23 & 23 solt 100 p. 100 \\
\hline ziguey ${ }^{+}$ & 33 & 32 solt $96,96 \mathrm{p} \cdot 100$ \\
\hline Abëché & 96 & 96 soit $100, \quad$ p. 100 \\
\hline Fort-Lamy & 73 & 73 soit 1000 P. 100 \\
\hline Total & 559 & 555 solt 99,2 日. 100 \\
\hline
\end{tabular}

+ = Animaux falsant partie d'un même troupeau (p̣elotons mêharıstes). 
TABLEAU $\mathrm{N}^{\circ}$ II

Titrage des antlcorps par Inhibftion de I'hêmagglutination.

\begin{tabular}{|c|c|c|c|c|c|c|c|c|c|c|c|}
\hline \multirow{2}{*}{$\begin{array}{l}\text { Nombre de sêtums } \\
\text { Lacalitếs }\end{array}$} & \multicolumn{11}{|c|}{ Titres des sêrums } \\
\hline & Négatifs & $1 / 1$ & $1 / 2$ & $i / 4$ & $1 / 8$ & $\begin{array}{c}\text { Positifs } \\
1 / 10\end{array}$ & à : $1 / 20$ & $1 / 40$ & $1 / 80$ & $1 / 160$ & $1 / 320$ \\
\hline $\begin{array}{c}\text { Moussoro } \\
\text { Lot } 1: 79 \\
\text { (reste } 5 \text { B sêrums } \geqslant 1 / 10 \text { ) }\end{array}$ & 0 & 5 & 7 & 13 & B & 4 & 10 & 21 & 7 & 2 & 2 \\
\hline $\begin{array}{l}\text { Moussoro } \\
\text { Lot } 2: 63\end{array}$ & 0 & 0 & 0 & 0 & 0 & 8 & 22 & 23 & 4 & 3 & 3 \\
\hline $\begin{array}{c}\text { Mao : } 120 \\
\text { (reste } 14 \text { sérums } \geqslant 1 / 10)\end{array}$ & 3 & 9 & 7 & 6 & 2 & 13 & 18 & 30 & 27 & 3, & 2 \\
\hline Nokou : 23 & 0 & 0 & 0 & 1 & 3 & 5 & 4 & 7 & 1 & 1 & 1 \\
\hline Zigurey : 33 & 1 & 2 & 4 & 2 & 7 & 3 & 5 & 5 & 2 & 1 & \\
\hline $\begin{array}{c}\text { Abëchê : } 55 \\
\text { (reste } 41 \text { sérums } 1 / 10 \text { ) }\end{array}$ & 0 & 5 & 2 & 10 & 10 & 10 & 8 & 7 & 2 & 1 & \\
\hline $\begin{array}{c}\text { Fort-Lamy : } 16 \\
\text { (reste } 57 \text { sêrums } \geqslant 1 / 10)\end{array}$ & 0 & 6 & 5 & I & 3 & & & 1 & & & \\
\hline 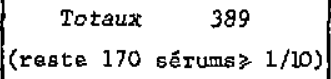 & 4 & 27 & 25 & 51 & 33 & 43 & 67 & 94 & 43 & 11 & 8 \\
\hline
\end{tabular}

TABLEAU N" III

Valeur moyenne des résultats obtenus en électrophorèse.

\begin{tabular}{|c|c|c|c|c|c|c|c|}
\hline & $\begin{array}{l}\text { Proteines } \\
\text { totales }\end{array}$ & Albumine & \multicolumn{4}{|c|}{$\begin{array}{c}1 \circ \mathrm{o} b \\
\alpha 2\end{array}$} & $\frac{\text { Albumine }}{\text { Globulines }}$ \\
\hline Sérums négatifs & $71,25 \mathrm{~g} / 1$ & 35,8 & 6,6 & 11,2 & 13,2 & 33,3 & 0,55 \\
\hline Séruma $<1 / 10$ & $70,05 \mathrm{~g} / 1$ & 35,4 & 8,0 & 9,0 & 13,3 & 34,3 & 0,54 \\
\hline Sêruma $>1 / 10$ & $75,78 \mathrm{~g} / 1$ & 33,8 & 6,3 & 9,3 & 14,2 & 36,4 & 0,51 \\
\hline Moyenne & 72,36 & 35,0 & 7,0 & 9,8 & 13,5 & 34,7 & 0,53 \\
\hline Kchouk et Durand (7) & $83,6 \pm 2,8$ & $43,5 \pm 4$ & $4,8 \pm 1,2$ & $9,3+2$ & $17,2 \pm 1,5$ & $27,3 \pm 3$ & 0,70 \\
\hline Holler et Hasian ( 6 ) & $83,1 \pm 0,85$ & $40,7 \pm 4,5$ & $11,8 \pm$ & 2,3 & $17,1 \pm 2,1$ & $30,3 \pm 6$ & 0,68 \\
\hline $\begin{array}{l}\text { Queval, Graber et } \\
\text { Bqunet (13) }\end{array}$ & 80,5 & 37,0 & 7,4 & 9,4 & 18,2 & 28,0 & 0,55 \\
\hline
\end{tabular}

\section{DISCUSSION}

1) If n'a pu être mis en évidence dans les sérums de dromadaires d'inhibiteurs non spécifiques dans l'hémagglutination par Myxovirus parainfluenzo 3.

2) L'incidence des anticorps inhibant l'hémagglutination par Myxovirus parainfluenza 3 est singulièrement élevée chez le dromadaire du
Tchad, pratiquement 100 p. 100 , ce qui signe que l'infection cameline à virus para 3 est largement répandue dans les troupeaux de dromadaires des régıons sahéliennes de ce pays. Les résultats obtenus quant au pourcentage d'infection, se rapprochent de ceux obtenus chez les bovins d'Afrique Centrale par PROVOST ef coll. (11) ce qui n'a rien d'étonnant quand on connaît les contacts existant entre 
zébus et dromadarres des régions intéressées par cette enquête. On peut se demander si l'infection à virus paraınfluenza 3 dans l'espèce cameline correspond à un virus adapté à elle ou à des souches bovines. Dans cette optique, it serait particulièrement intéressont d'analyser les sérums de dromadaires des régions sahariennes du Tchad (Largeau, Fada) où se pratique seulement l'élevage du chameau, à l'exclusion de celui des bovins et ovins.

3) On peut également faire les constatations suivantes :

Les titres observés sont parfois peu élevés : 27 sérums positifs purs, 25 à la dilution du 1/2, 51 au 1/4, 33 au $1 / 8$ (tableau II).

- Dans certains cas ils sont relativement élevés : 43 sérums positifs à la dilution du 1/80, 11 au 1/160, 8 au 1/320 (tableau Ii).

Il est logique de penser que suivant le cas, ces sérums correspondent à une affection ancienne (faible titre d'anticorps) ou à une infection récente ou du molns une réinfection (titre élevé d'anticorps).
- Les réponses obtenues dans certains troupeaux (peloton méhariste de Moussoro) sont homogènes : 63 sérums $\geqslant 1 / 10$ sur 63 exemplaires (tableau II), ce qui laisse supposer que l'infection à virus parainfluenza 3 peut évolver de façon épizootique à l'intérieur du même troupeau, à l'âge adulte et peut être ainsi un agent infectieux d'importance non négligeable.

4) Tout récemment, SINGH et coll. (14) n'ont noté en Egypte à l'abattoir du Caire que deux sérums de dromadares positifs sur 52 analysés (soit 3,8 p. 100) ef 10 sérums d'ovins positifs sur 64 analysés (soit 15,6 p. 100). Le pourcentage d'infection à virus parainfluenza 3 semble donc bien plus élevé chez le dromadaire des régions sahéliennes du Tchad que chez ceux d'Egypte. Rappelons à ce sujet que CHERBY et coll. (1), lors d'une enquête sımilaire chez des bovins en France, viennent de montrer que l'influence de la région est statistiquement déterminante dans les réponses obtenues. II semble bien que cette constatation soit également vérıfiée sur le continent africain en ce qui concerne les dromadaires.

\section{SUMMARY}

Survey in Parainfluenza 3 virus infection in dromedary in Chad

The incidence of parainfluenza 3 virus infection in dromedary in Chad has been studied using the haemagglutination inhibition test carrled out on 559 serum samples. The results indicated that this infection was widespread in the Sahelian area of this country. The infection is likely to occur in epizootic form inside a herd, but without any clinical sign.

The dromedary sera did not appear to include non-specinic inhibition of the parainfluenza 3 virus haemagglutimation. The sera which showed negative reactions to this inhibition test were not from hypo-or agammaglobulinemic animals.

\section{RESUMEN}

Encuesta sobre la infeccion con el virus parainfluenza 3 en el dromedario de Chad

Los autores estudiaran la incidencia de la infección con el virus parainfluenza 3 en el dromedario de Chad, utilizando latécnica de ınhibición de hemaglutinación para el analisis de 559 sueros. Los resultados demuestran que, muy a menudo, se encuentra la dicha infección en las regiones sahelıanas de éste país. A lo que parece, la enfermedad ocurre bajo forma epizoolica en una manada, pero sin signo clínico. 


\section{BIBLIOGRAPHIE}

1. CHERBY (J.), PETERMANN (H. G.), BERANGER (G.) et SOULEBOT (J. P.).-Enquête sérologique chez les bovins en France; recherche des anticorps inhibant l'hémagglutination due à Myxovirus parainfluenza 3. Rec. Méd. vét., 1967, 143 : 755-765.

2. DAWSON (P. S.). - The nature of substances present in normal bovine sera inhibiting the activity of Porainfluenza 3 virus. J. Comp. Poth., 1963, $73:$ 428-436.

3. FAYE (P.), CHARTON (A.) et LE GAYEC (Cl.). - Présence dans le sérum d'ovins d'anticorps inhibant l'hémagglutination par Myovirus parainfluenza 3. Bull. Acad. vét., 1967. $40: 203-207$.

4. FLEURY (P.). - Fiches techniques de chimie biologique. Paris, Vega, 1946.

5. HAMPARIAN $(V, V$.), WASHKO $(F, V$. $)$, KETLER (A.) et HILLEMAN (M. R.). Laboratory and field investigations of bovine Myxovirus parainfluenza 3 virus and vaccine. III. Evaluation of an SF4 (shipping fever) virus vaccine in cattle. J. Immun., 1961, 87: 139-146.

6. HOLLER $\left(H_{1}\right)$ and HASSAN (M.). - Bestimmung einiger blutbestandteile bei kamelen im Sudan. Dt. tierärztl. Wschr., 1966, 73 : 553-556.

7. KCHOUK (M.), DURAND $(M$.$) . - Quel-$ ques dosages chimiques dans le sang des dromadaires en Tunisie. Arch. Inst. Pasteur Tunis, 1958, 35 (1) : 3-37.

8. KETLER (A.), HAMPARIAN (V. V.). HILLEMAN (M. R.) et WASHKO (F.V.).-- Laboratory and field investigations of bovine
Myxovirus parainfluenza 3 virus and vaccine. I. Properties of the SF4 (shipping fever) strain of virus. J. Immun., 1961, 87 : 126-133.

9. NGUYEN-BA-VY et PERREAU (P.). - Existence d'anticorps spécifiques de Myxovirus parainfluenzo type 3 chez les bovins de France. Rec. Méd. Vét., 1967, 143 : 245-251.

10. PROVOST (A.), BORREDON (C.) et QUEVAL $\left(R_{1}\right)$. - Une hypogammaglobulinémie essentielle des bovins d'Afrique centrale, cause d'erreur dans les enquêtes sérologiques. Rev. Elev. Méd. vét. Poys trop., 1965, $18: 385-393$.

11. PROVOST (A.), BORREDON (C.), QUEVAL (R.) et MAURICE (Y.). - Enquête sur l'infection des bovidés par le virus parainfluenza 3 en Afrique centrale. Application du contrôle de la sérologie de la péripneumonie. Rev. Elev. Méd. vét. Pays trop., 1967, 20 : 51-59.

12. QUEVAL (R.). - Contribution à l'étude des protéines sériques du zébu arabe au Tchad. Rev. Elev. Méd. vét. Pays trop., 1959, 12 : 293296.

13. QUEVAL (R.), GRABER (M.) et BRUNET (Mme). - Etude de la protidémie et des constantes hématologiques des camélidés en fonction des helminthes dont ils sont porteurs. Rev. Elev. Méd. vét. Poys trop., 1967, 20, 437449.

14. SINGH (K. V.) et ATA (F. A.). - Presence of antibodies against parainfluenza 3 virus. in camel and sheep sera. Vet. Rec., 1967, 81 : 84. 